\title{
A Study on Turbulent Flow and Heat Transfer of an Off-set Jet
}

\author{
Dr. Ramesh Chandra Mohapatra \\ Government College of Engineering, Biju Patnaik University \& Technology (BPUT) \\ Department of Mechanical Engineering, (BPUT), Orissa, India
}

\begin{abstract}
One most common $\kappa-\varepsilon$ model is used for study of the turbulence in the off-set jet problem. Finite Volume based SIMPLE algorithm is used for solving the discretized equations and power-law scheme is used for discretizing the convection terms The flow and heat transfer characteristics in the wall jet cooling that had already been done experimentally, is also shown. The regions of study are the recirculating zone, impingement and developing wall jet zones. Thermal characteristic of each zone is studied. The numerical results are compared with the experimental results. Computational details of various mean flow and turbulent parameters are presented and described for different offset ratios. The results include reattachment length, velocity profile, the axial velocity decay curve and wall static pressure distribution. The spatial distributions of turbulent intensities show a high turbulent recirculating flow between the jet and the off-set plate. The results obtained are satisfactory with the experimental results.
\end{abstract}

Keywords: Off-set jet, $\kappa-\varepsilon$ model, Power law scheme, Recirculating zone, Off-set ratio, Reattachment length

\section{INTRODUCTION}

An off-set jet occurs when discharge of fluid, in the form of jet, occurs in the proximity of surface such that the surface influences the entrainment charecteristics asymmetrically about the jet centre line. The most obvious effects is the derection of the jet towards the wall and eventually impingement onto the surface. The off-set jet commonly represents the flow like heat exchangers, fluid injectors, environmental dischargers, combustion chambers, cooling systems and many others. When the height (h) $=0$, the off-set jet becoms a wall jet. Asymmetric entrainment on both sides of the jet causes the jet to deflect towards the plate and finally attaches to it. The jet is divided into three regions, viz, the recirculation region, the impingement region and the wall jet region. The details of the flow was studied by several authors. The first detail experimental study of the mean flow was reported by Bourque and Newman [1], followed by Sawyer [2]. They have examined the effect of off-set height on the wall pressure distribution and reattachment locations. Rajaratnam and Subramanya [3] found that beyond the impingement region the flow represents similar to a wall jet flow irrespective of the off-set ratio. Hoch and Jiji $[4,5]$ have considered the case of an off-set jet in the presence of a free stream motion. The fluid flow solution has been provided by them. Utilizing this fluid flow solution, they later on have provided the analytical solution for the temperature distribution for the same geometry. In a study, Pelfrey and Liburdy [6] have provided the details of the mean flow and turbulent characteristics and showed how entrainment and local pressure and turbulent energy components are affected by the jet curvature in the reattachment region. In this study, the plate parallel to the jet was as adiabatic. For large Reynolds number generally larger than the $10^{4}$ the impingement distance becomes a function of the off-set ratio only. Holland and Liburdy[7] presented the thermal characteristics of off-set jets in a condition similar to that of Pelfrey and Liburdy for different offset ratios. In the study, they examined the surface temperature distribution, the maximum temperature decay and the temperature variation in three regions. Computations of the off-set jet performed by Yoon et al[8] showed that in addition to the turbulence model. The numerical scheme also plays a profound effect in predicting the solution. It is observed that the numerical diffusion error due to the stream line to grid-skewness will be significant in the prediction of off-set jet flows. Koo and Park [9] have used the QUICKER scheme to predict the turbulent off-set jet flows. They have compared their numerical results with those from the skew-upwind and the hybrid schemes. They concluded that the QUICKER scheme is found to be suerior to the other two schemes. Nasr and Lai['10] have done an experimental study for the offset ratio(OR) $=2.125$ using laser doppler 
anemometry (LDA).The LDA results had been used to examine the capability of three different turbulence models (i.e, $\kappa-\varepsilon$; RNG, and Reynolds stress) in predicting the velocity field of this jet. It was found that although three turbulence models predict quantitatively, the standard $\kappa-\varepsilon$ turbulence model predicts better the reattachment length with experimental value. Yang and Shyu[11] have presented numerical prediction on fluid flow and heat transfer characteristics of multiple impinging slot jets with an inclined confinement surface. The parameters studied included the angle of inclined confinement surface and entrance Reynolds number, which is in the turbulence region. The numerical results showed that the maximum local Nusselt number and maximum pressure on the impinging surface move downstream while the inclination angle is increased. The maximum local Nusselt number decreases while the value of the local Nusselt number downstream increases with increasing inclination angle. The entrance Re has little effect on the size of the recirculation region, but the inclination angle has the significant effect on the recirculation zones. Shuja et al. [12] studied the jet impingement on a surface having constant heat flux over a limited area. Air is considered as the impinging gas and the process is simulated with a two dimensional axis symmetric form of the governing conservation equations. Four turbulence models including standard $\kappa-\varepsilon$, low Reynolds number $\kappa-\varepsilon$, and two Reynolds stress models, are introduced to account for the turbulence. They concluded that the agreement between the temperature profiles predicted from both the low Reynolds number $\kappa-\varepsilon$ model and the Reynolds stress turbulence model are better than that obtained from the standard $\kappa-\varepsilon$ model. Aldabbagh and Sezai[13] have investigated numerically the steady state flow and heat transfer characteristics of impinging laminar square twin jets. The simulations have been carried out for various jet-to-jet spacing and nozzle exit-to-plate distances. The effect of these parameters on the flow structure has been studied. Heat transfer from a row of turbulent jets impinging on a stationary surface is investigated numerically by Salamah and Kaminski [14]. The jetto-jet interaction, the geometric parameters of the jet array, and the effect of Reynolds number are investigated. The conjugate heat transfer of a laminar off-set jet is reported by Kanna and Das [15]. The governing equation is solved by alternating direction implicit (ADI) method and clustered Cartesian grids are used for computations. They found that the local Nusselt number increases to a peak value and further it is decreased in the downstream direction. In another study, the same authors have carried out a steady-state heat-transfer study for a two dimensional, laminar, incompressible, plane wall jet over a backward facing step. The heat transfer characteristics of the jet as functions of Reynolds number, Prandtl number, and step geometry (step length and step height) are reported in detail. Results are presented in the form of isotherm, Nusselt number, and average Nusselt number. In some cases, the computed results are compared with the results when the step length is zero. Despite the wide variety of engineering applications, efforts to numerically predict the turbulent off-set jet flows and heat transfer have been rare. In the present study, computational details of the various offset ratios (wall jet, $\mathrm{OR}=7$, and 11) are presented. Power law scheme is used for discretizing the convective terms. In order to have the same numerical accuracy for the fluid flow solution as reported in Koo and Park, high grid size is used and non- uniform grids are used along the walls and at the entrance of the jet. The geometry is similar to that of Pelfrey and Liburdy. The results are compared and are found to be in good agreement with it. For the temperature solution, the case of Holland and Liburdy has been considered. The computed results have been compared and found to be in good agreement with the experimental results.

\section{Problem Specification}

The flow field of an off-set jet is complex and is encountered in many engineering applications. A jet can be analyzed as a free jet or bounded jet, depending on distances from the discharge to the confining boundaries. If the boundaries are sufficiently far from the discharge, the flow can be considered as free jet. However, a bounded jet will occur when it interacts with a parallel wall. Bounded jet can be classified into three types; (a) impinging jet aimed towards the boundary; (b) wall jet where fluid is discharged at the boundary; and (c) off-set jet from a vertical wall of a stagnant pool issuing parallel to a horizontal solid wall. The flow emanating from a two dimensional (2D) off-set jet is shown in (Fig.1). where the main features and regions of interest are depicted. Fluid is discharged from a slot in a vertical wall into the ambient near a horizontal solid boundary parallel to the inlet jet direction. Due to the entrainment of fluid between the jet and the bottom plate, there is a reduction of pressure in this region forcing the jet to deflect towards the boundary and eventually attach with it. This is called Coanda effect. The off-set jet behaves differently in various regions. In the near field with in a very short distance from the point of discharge, the jet in momentum dominated flow having 
the properties of free jet. Attachment may occur when the jet is deflected by an adjacent solid wall or a free surface and tends to flow along the boundary. In the region around attachment point, that is, the impingement region and part of pre- attachment region, the jet has characteristics similar to an impingement jet. The jet becomes a wall jet in the far field. The other factors such as ambient motion (free- stream velocity), buoyancy (density difference), discharge orientation, etc. further complicate the jet boundary interaction and the behavior of an off-set jet. The turbulent phenomenon of the jet is studied using the $\kappa-\varepsilon$ model.

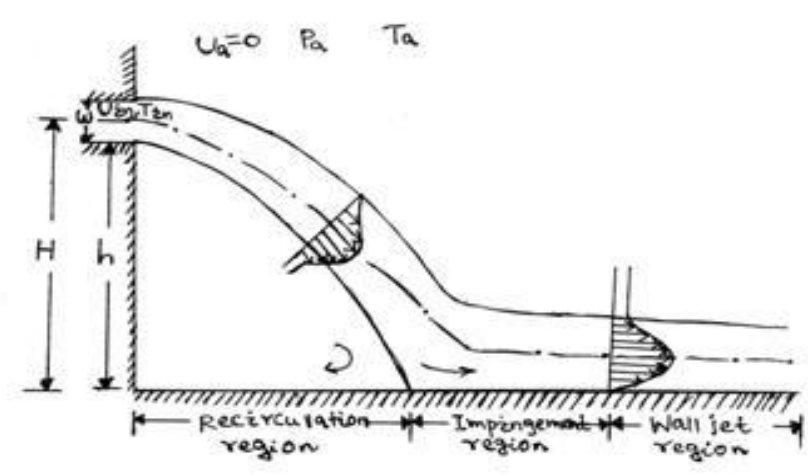

Fig1. Flow through off-set Jet.

\section{Governing EquATIONS}

Continuity equation

$$
\frac{\partial \bar{U}}{\partial x}+\frac{\partial \bar{V}}{\partial y}=0
$$

$\mathrm{X}$ - Momentum equation

$$
\frac{\partial(\bar{U})^{2}}{\partial x}+\frac{\partial(\overline{U V})}{\partial y}=-\frac{1}{\rho} \frac{\partial \bar{P}}{\partial x}+\frac{\partial}{\partial x}\left[\left(v+v_{t}\right) \frac{\partial \bar{U}}{\partial x}-\frac{2}{3} \kappa\right]+\frac{\partial}{\partial y}\left[\left(v+v_{t}\right) \frac{\partial \bar{U}}{\partial y}\right]
$$

Y- Momentum equation

$$
\frac{\partial(\overline{U V})}{\partial x}+\frac{\partial(\bar{V})^{2}}{\partial y}=-\frac{1}{\rho} \frac{\partial \bar{P}}{\partial y}+\frac{\partial}{\partial x}\left[\left(v+v_{t}\right) \frac{\partial \bar{V}}{\partial x}\right]+\frac{\partial}{\partial y}\left[\left(v+v_{t}\right) \frac{\partial \bar{V}}{\partial y}-\frac{2}{3} \kappa\right]
$$

Energy equation

$$
\frac{\partial(\overline{U T})}{\partial x}+\frac{\partial(\overline{V T})}{\partial y}=\frac{\partial}{\partial x}\left[\left(\alpha+\alpha_{t}\right) \frac{\partial \bar{T}}{\partial x}\right]+\frac{\partial}{\partial y}\left[\left(\alpha+\alpha_{t}\right) \frac{\partial \bar{T}}{\partial y}\right]
$$

Turbulent kinetic energy $(\kappa)$ equation

$$
\frac{\partial[(\bar{U}) \kappa]}{\partial x}+\frac{\partial[(\bar{V}) \kappa]}{\partial y}=\frac{\partial}{\partial x}\left[\left(v+\frac{v_{t}}{\sigma_{\kappa}}\right) \frac{\partial \kappa}{\partial x}\right]+\frac{\partial}{\partial y}\left[\left(v+\frac{v_{t}}{\sigma_{\kappa}}\right) \frac{\partial \kappa}{\partial y}\right]+G-\varepsilon
$$

Rate of dissipation $(\varepsilon)$ equation

$$
\frac{\partial[(\bar{U}) \varepsilon]}{\partial x}+\frac{\partial[(\bar{V}) \varepsilon]}{\partial y}=\frac{\partial}{\partial x}\left[\left(v+\frac{v_{t}}{\sigma_{\kappa}}\right) \frac{\partial \varepsilon}{\partial x}\right]+\frac{\partial}{\partial y}\left[\left(v+\frac{v_{t}}{\sigma_{\kappa}}\right) \frac{\partial \varepsilon}{\partial y}\right]+c_{1 \varepsilon} \frac{\varepsilon}{\kappa} G-c_{2 \varepsilon} \frac{\varepsilon^{2}}{\kappa}
$$


Production term $(\mathrm{G})$

$G=v_{t}\left[2\left(\frac{\partial \bar{U}}{\partial x}\right)^{2}+2\left(\frac{\partial \bar{V}}{\partial y}\right)^{2}+\left(\frac{\partial \bar{U}}{\partial y}+\frac{\partial \bar{V}}{\partial x}\right)^{2}\right]$

Eddy Viscosity $\left(v_{t}\right)$

$v_{t}=c_{\mu} \frac{\kappa^{2}}{\varepsilon}$

Assumptions made are:-

a. Steady turbulence.

b. 2-D flow, flow variations in z- direction is neglected

c. Incompressible flow, i.e. dilation term is neglected.

d. Body forces are neglected.

e. Properties $(\mu, v, \rho$,$) are assumed to be constant.$

f. RANS equations are used for predicting the turbulent flow.

g. Boussinesq assumption is used to link Reynolds stress to velocity gradient.

h. $\kappa-\varepsilon$ Model is used for determining the turbulent viscosity.

\section{BOUNDARY CONDITIONS}

Non dimensional boundary conditions are provided as input for the solution. At the inlet of the jet $\overline{U_{n}}=\frac{\bar{U}}{U_{0}}=1, \overline{V_{n}}=\frac{\bar{V}}{V_{0}}=0$ and $\theta=\frac{T-T_{\infty}}{T_{i n}-T_{\infty}}=1$ are the boundary conditions for velocity and temperature. For turbulent kinetic energy equation, boundary conditions at inlet is $\kappa_{n}=\frac{\kappa}{U_{0}{ }^{2}}=1.5 I^{2}$ where I is equal to turbulent intensity, and is equal to 0.02 . For solid wall no slip boundary condition is considered for velocity. For bottom surface adiabatic surface is considered and for vertical surface isothermal surface is considered. First grid point lies at point $\mathrm{P}$ in the logarithmic region. There are still five unknown constants $c_{1 \varepsilon}, c_{2 \varepsilon}, \sigma_{\varepsilon}, \sigma_{\kappa}, c_{\mu}$ which are universal for all types of flow. Experimental data are used to determine the constants. The value $c_{\mu}=0.09$. For determining the other constants logarithmic part of turbulent boundary layer is considered where convection term is negligible, and utilizing that production and dissipation are in balance. $P_{\kappa}=\rho \varepsilon$. The two constants $\sigma_{\kappa}, \sigma_{\varepsilon}$ are optimized by applying the model to various fundamental flows such as flows in channel, pipes, jets, wakes, etc. The values of the five constants $c_{1 \varepsilon}, c_{2 \varepsilon}, \sigma_{\varepsilon}, \sigma_{\kappa}, c_{\mu}$ are 1.44, 1.92, 1.31, 1.0 and 0.09 respectively.

\section{Code VAlidation}

To validate the code developed, the results obtained from code are compared with the experimental solution by Holland and Liburdy [7] for offset ratio $=7$ shown in Fig. (2). To do this, velocity profiles are compared at different downstream location, that shows the little difference in the deviation from the experimental results. These downstream locations lie in the different region. Downstream location at $X=3$ (recirculating region), $X=6$ (recirculating region), $X=9$ (impingement region), $X=12$ (impingement region), $X=15$ (wall jet region) are considered. A grid independence study is done for grid sizes of $75 \times 61,101 \times 81,121 \times 101$ and 151x101.It is found that the grid size of 151x101 and the domain size of $75 \times 35$ is satisfactory for all the cases. Hence the grid size $151 \times 101$ is considered for all the cases. 


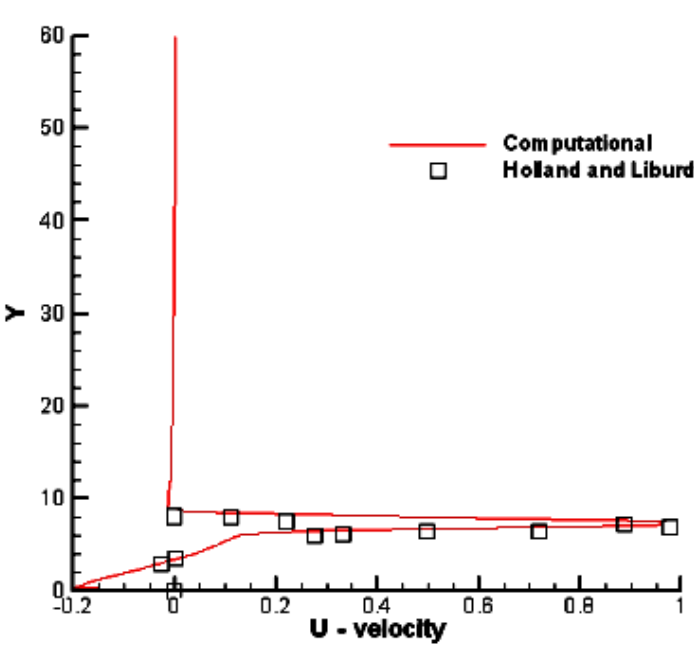

Fig2. (a) $X=3$

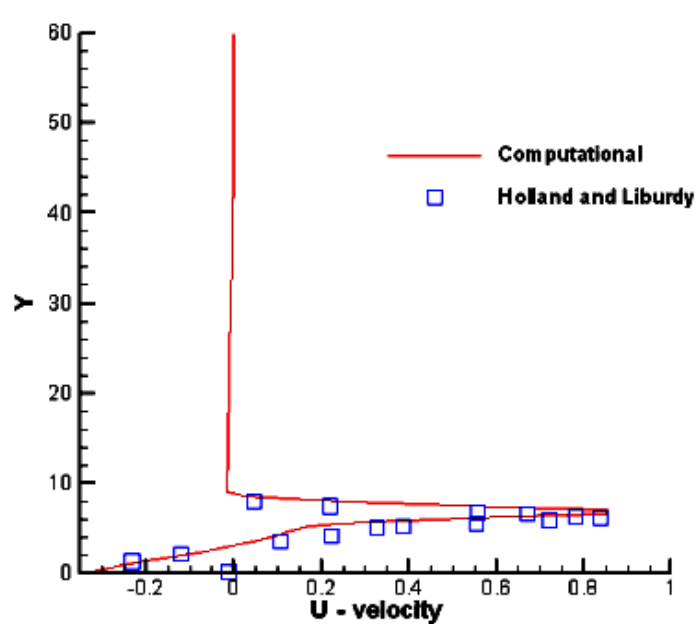

Fig2. (b) $X=6$

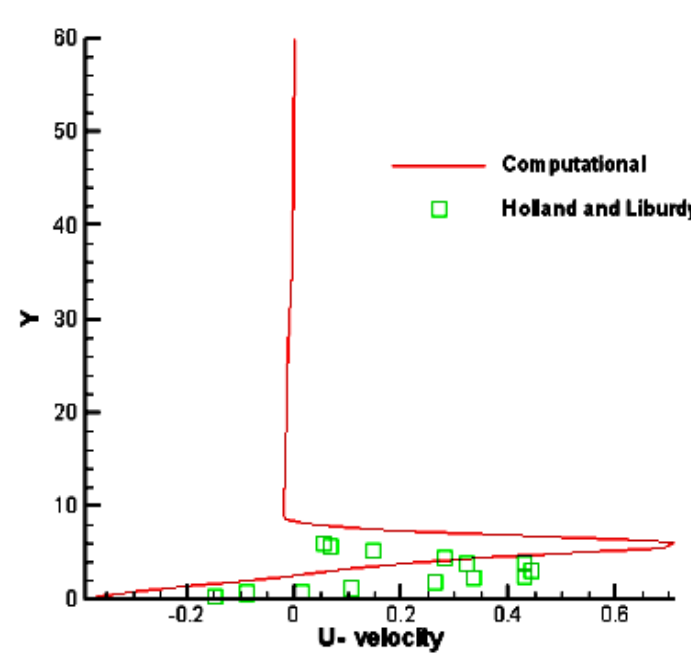

Fig2. (c) $X=9$

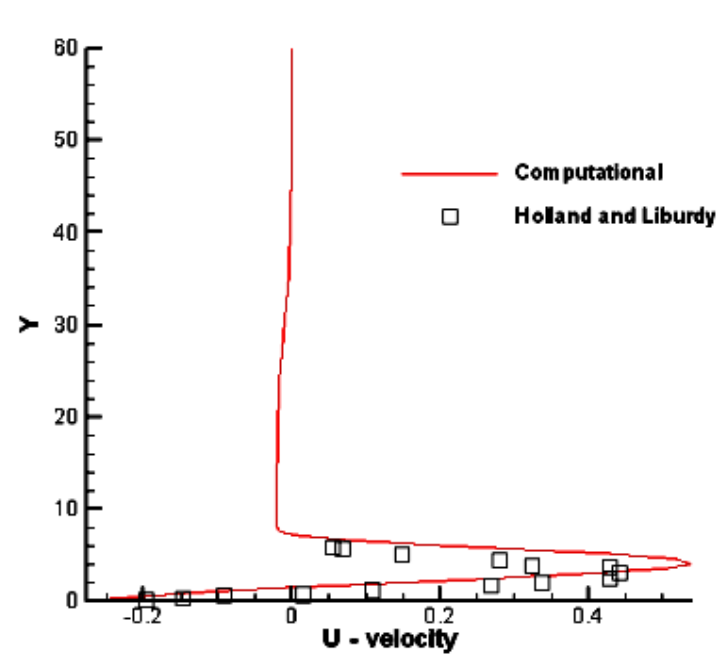

Fig2. (d) $X=12$

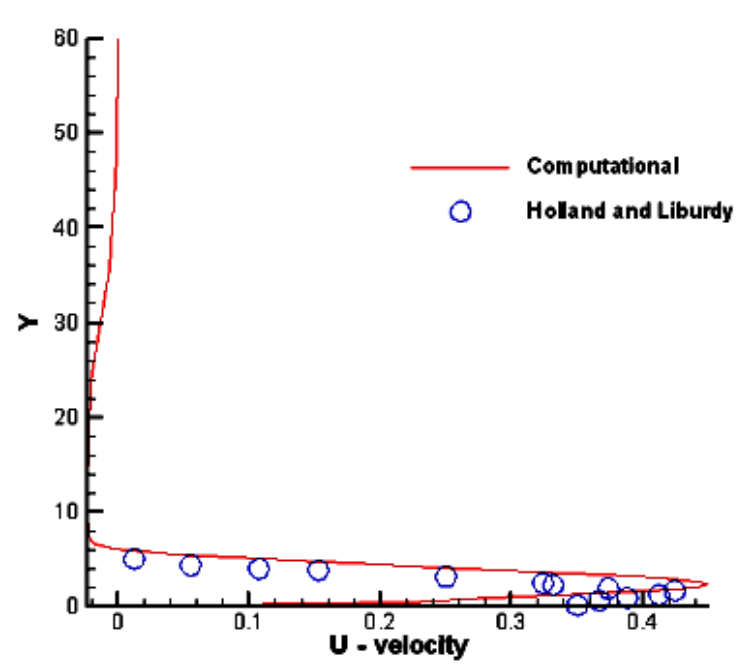

Fig2. (e) $X=15$

Figure2. Validation of code for O.R. $=7$ with experimental results of Holland and Liburdy

\section{RESUlTs \& DisCUSSIONS}

Code is run for offset ratios 7, 11 and wall jet .The grid size of $151 \times 101$ is considered for all cases. Here the working fluid is air and Reynolds number is 15000 . Following results are obtained from the code: 


\subsection{Reattachment Length}

This is the length from the offset wall to the point where the shear stress is zero. It's value increases as the offset ratio increases. For lower offset ratio the obtained values are lower than experimental values and higher for higher offset ratio shown in Fig (3). For higher offset ratios the results are quite satisfactory with the experimental values. This is due to numerical diffusion and applicability of the wall function in the region. The variations of reattachment length with offset ratio are shown in table 1 .

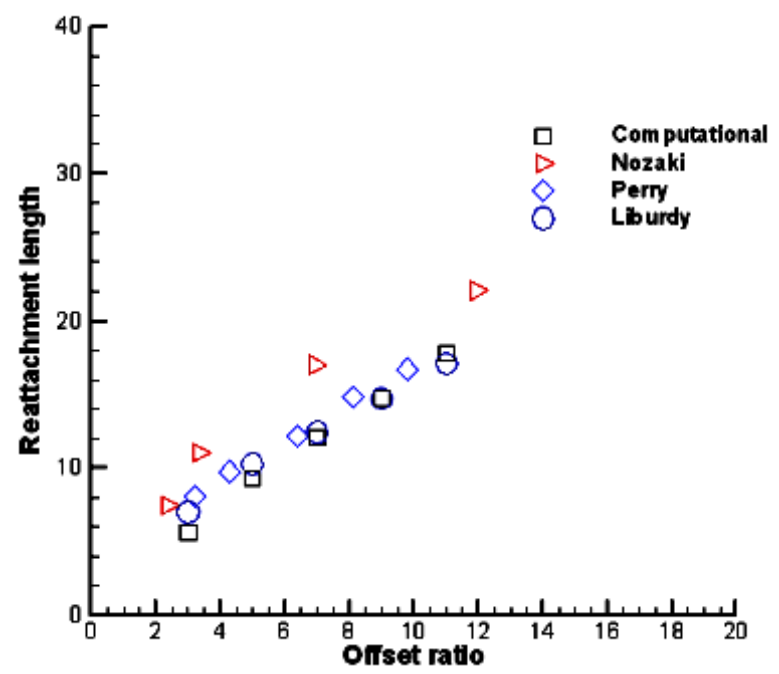

Figure3. Variation of reattachment length with Offset ratio

Table1. Variation of Reattachment length (R.L.) with offset ratio

\begin{tabular}{|c|c|c|}
\hline Offset ratio & R.L.( present solution) & R.L.(Experimental) \\
\hline 5 & 9.272 & 10.28 \\
\hline 7 & 10.067 & 12.42 \\
\hline 9 & 14.655 & 14.71 \\
\hline 11 & 17.842 & 17.10 \\
\hline
\end{tabular}

\subsection{Maximum Velocity Decay}

$\mathrm{U}_{\max }$ variation occurs in three regions. In the first region the maximum velocity decreases much faster up to reattachment region then there is sudden rise in the maximum velocity within the impingement region and slow drop in maximum velocity far from the downstream direction. In the decreasing region velocity drops slowly as the offset ratio increases shown in Fig.(4). Minimum value occurs very near to reattachment point and it decreases as O.R. increases. In the increasing region $U_{\max }$ increases slowly as the offset ratio increases. In the slowly decreasing region $\mathrm{U}_{\max }$ decreases as offset ratio increases at a particular location.

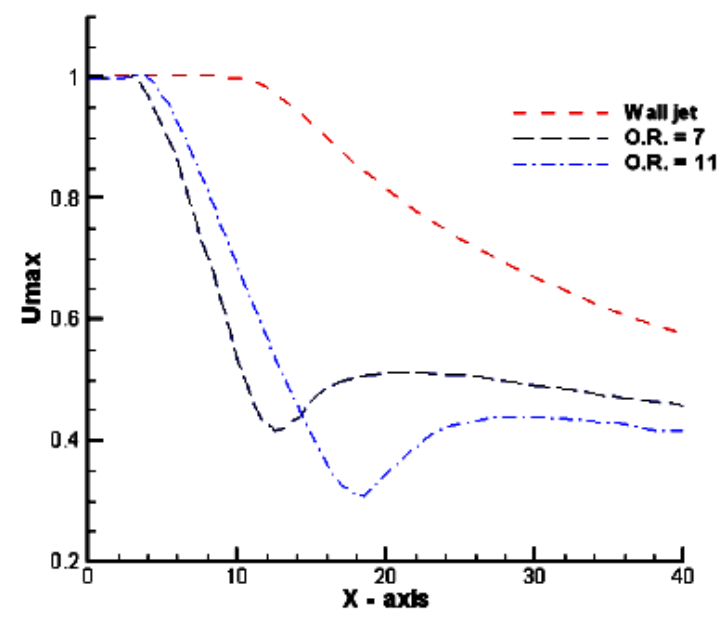

Figure4. Variation of maximum velocity decay with Offset ratio 


\subsection{Variation of Pressure at Wall}

Pressure attains a minimum value as the OR decreases because for low OR, jet impingement velocity is more. For the same reason wall pressure attains the maximum value as the OR increases. Minimum and maximum values of pressure are very close to center of vortex in the upstream direction in the recirculating region and downstream in the impingement region. Farther downstream pressure is almost constant and increases as offset ratio increases at any particular location shown in Fig.(5).

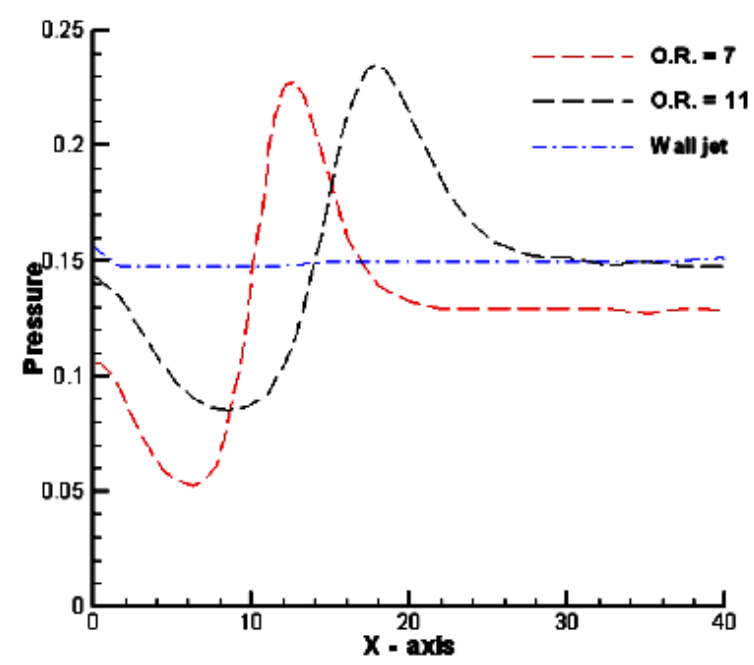

Figure5. Variation of wall pressure with offset ratio

\subsection{Half Velocity Points}

It is the vertical distance where the half of the maximum velocity occurs. For a particular X-coordinate there are two values one is the outer shear and other is inner shear layer. The distance $y_{+1 / 2}$ shows the region of outer shear layer and $\mathrm{y}_{-1 / 2}$ shows the inner shear layer. Fig(6) shows the spread of outer shear layer increases as the location $\mathrm{X}$ increases for a particular offset ratio. Spreading rate also increases with increase in offset ratio. Lower half velocity points have little variation with $\mathrm{X}$.
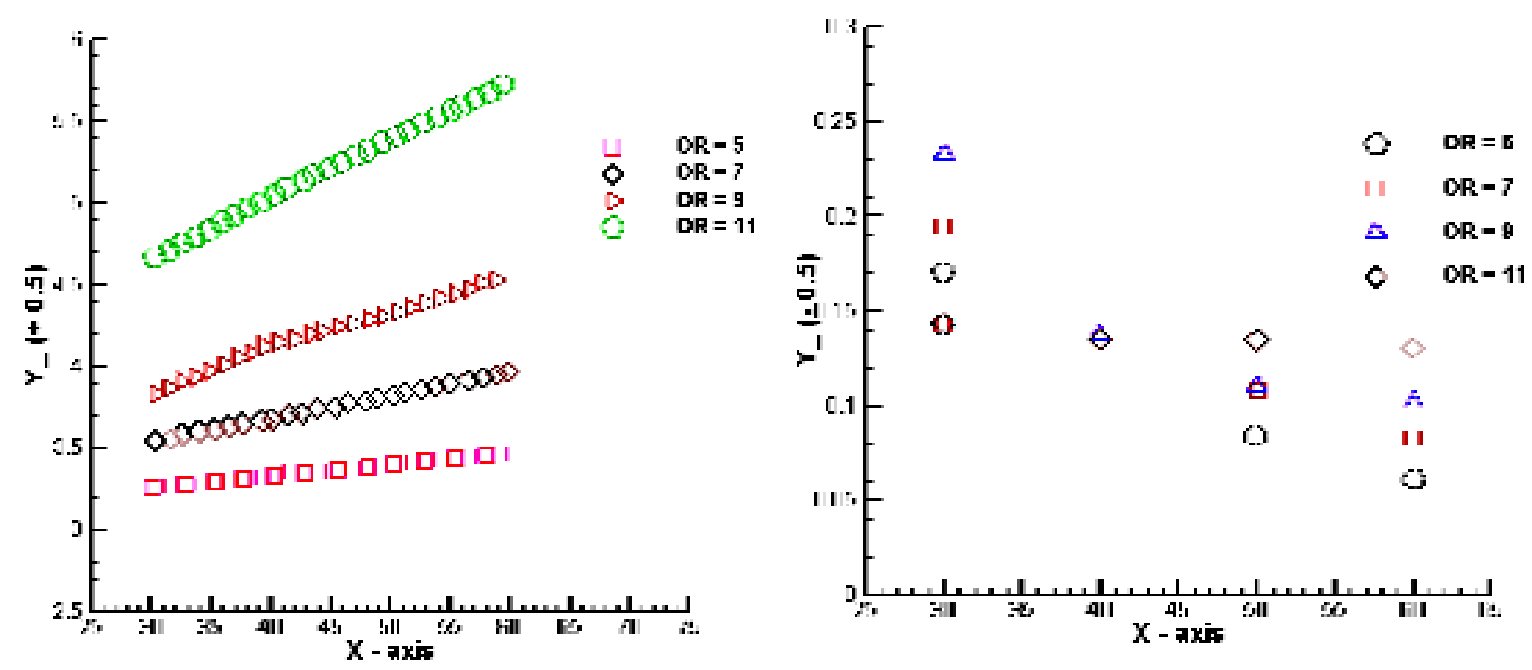

Figure6. Loci of half velocity points

\subsection{Stream Line Distribution}

Fig.(7) shows the stream line plot of the flow field. This figure is used for finding the reattachment length. The point of zero shear stress is called reattachment point. 


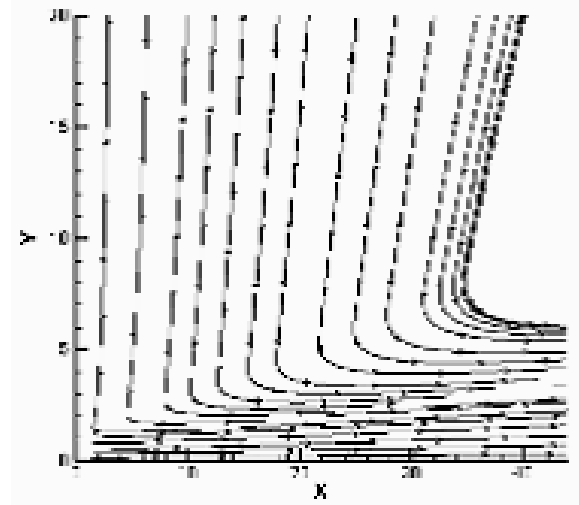

(a)

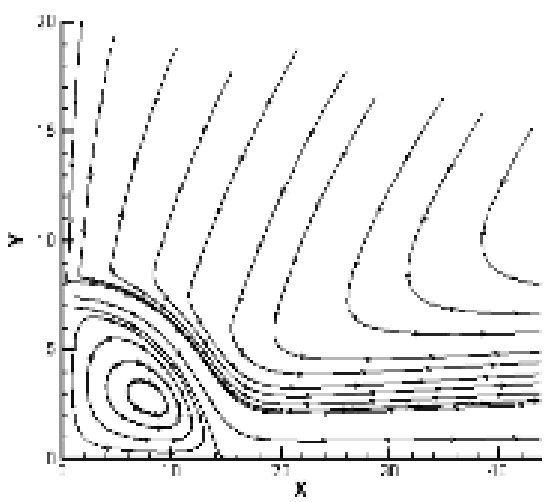

(b)

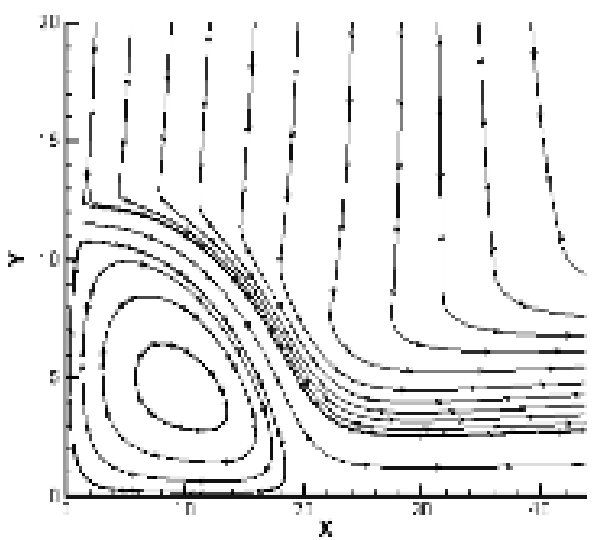

(c)

Figure7. Stream line distribution for (a) Wall jet (b) OR $=7$ and (c) OR $=11$

\subsection{Pressure Distribution in the Domain}

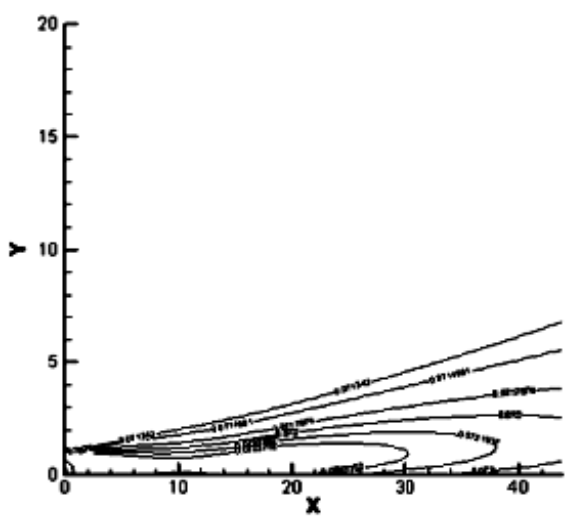

(a)

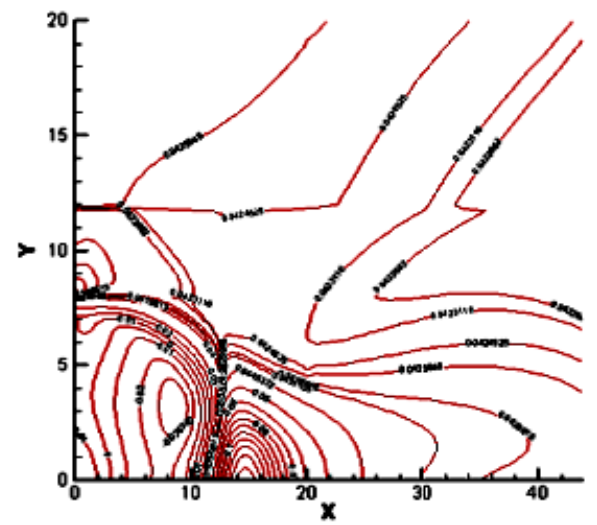

(b)

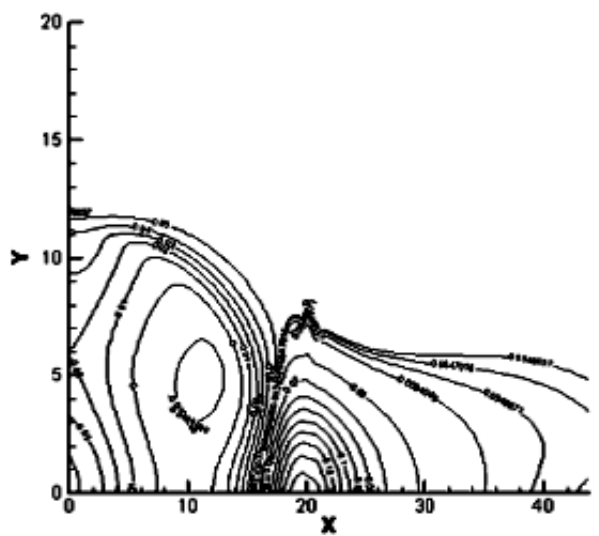

(c)

Figure8. Pressure distribution of Offset jet for (a) Wall jet (b) OR $=7$ and (c) OR $=11$ 
From the Fig( 8$)$ it is evident that variation of pressure in the recirculating and impingement region is high. Maximum and minimum values of pressure are found in impingement region and very close to center of vortex in the recirculating region respectively. At the impingement region fluid is brought to rest so the pressure at these points are higher and in the recirculating region due to entrainment of the fluid the sub atmospheric region creates where low pressure value exists.

\subsection{Distribution of turbulent kinetic energy $(\kappa)$}

Distribution of turbulent kinetic energy for offset jet with different offset ratios are shown in Fig(9). From this figure, it is obvious that maximum value of turbulent kinetic energy occurs in the recirculating region and in the nozzle exit region where it interacts with stationary fluid. In case of offset jet $\kappa$ is lower in the impingement region than recirculating region. Lower values are obtained in wall jet region. In the wall jet region $\kappa$ increases up to $U_{\max }$ and beyond that it decreases to almost zero.

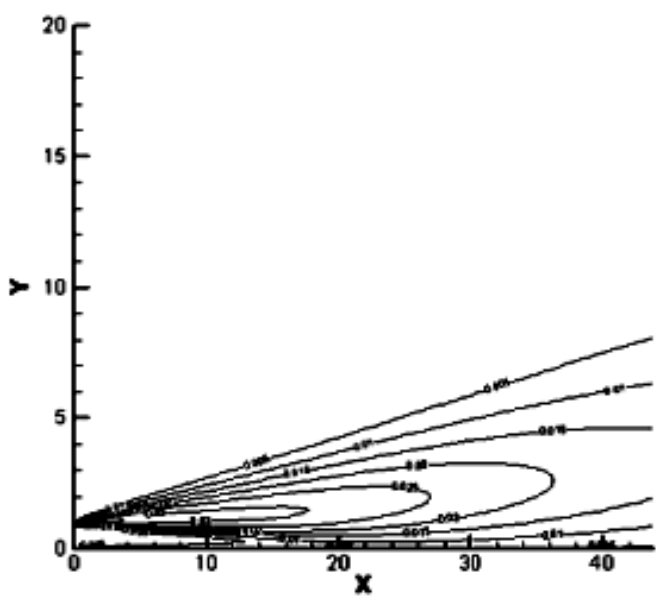

(a)

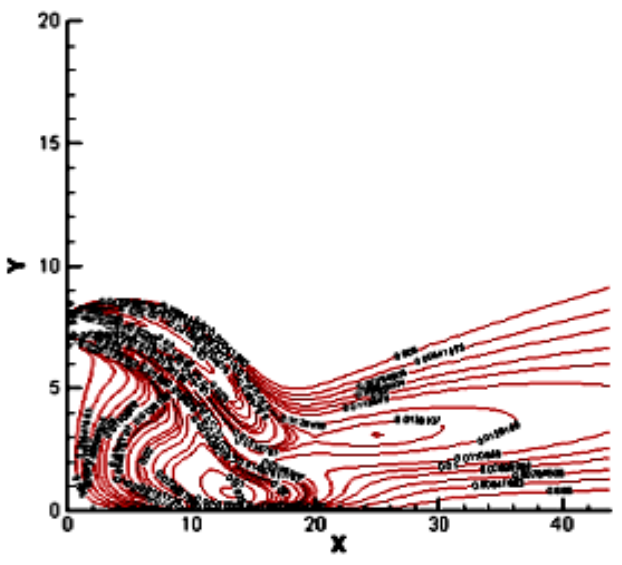

(b)

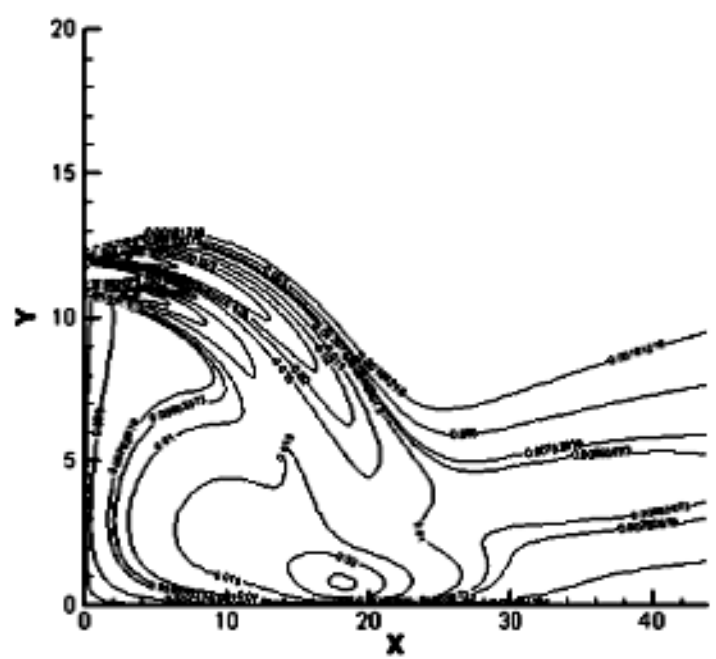

(c)

Figure9. Distribution of turbulent kinetic energy of Offset jet for (a) Wall jet (b) OR = 7 and (c) OR = 11

\subsection{Dissipation $(\varepsilon)$ of turbulent kinetic energy}

Maximum dissipation $(\varepsilon)$ occurs on either side of the jet. It has minimum value in the recirculating region. It decreases along the stream-wise direction. It is obvious from Fig(10). In the crosswise direction it increases up to $U_{\max }$ and then it decreases to zero. The value of dissipation suddenly drops to the zero at interaction of jet boundary to the ambient fluid. At nozzle exit higher value of dissipation observed with increase in offset ratio. 


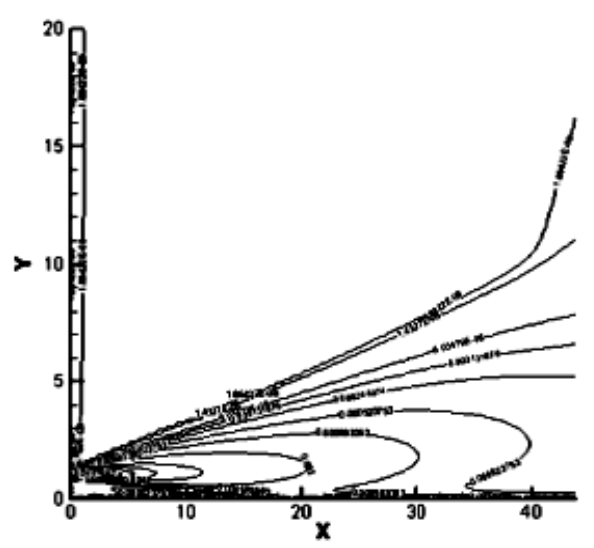

(a)

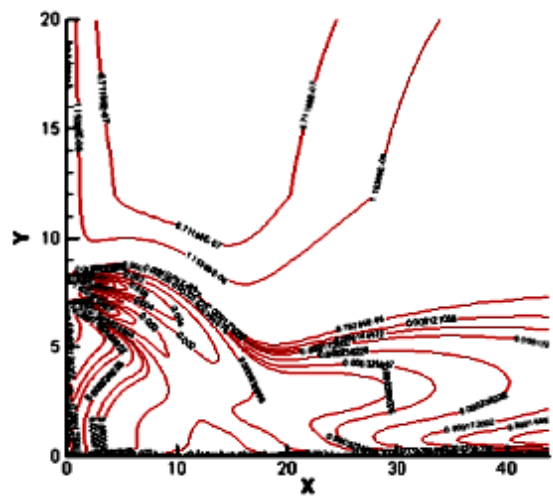

(b)

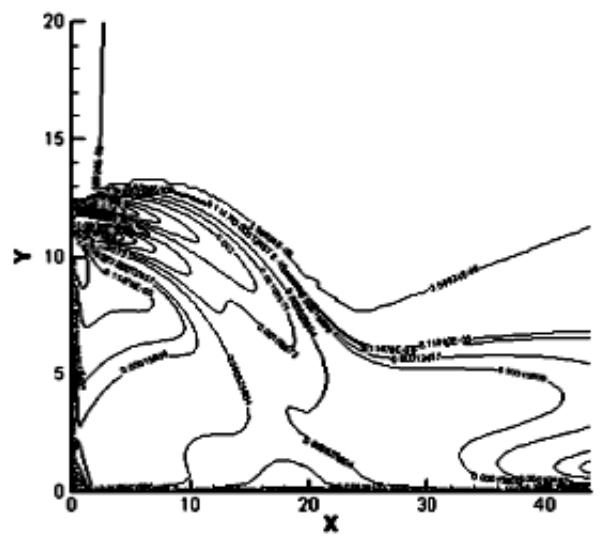

(c)

Figure10. Dissipation $(\varepsilon)$ of turbulent kinetic energy of Offset jet for $(a)$ Wall jet $(b)$ OR $=7$ and $(c)$ OR $=11$ 6.9. Distribution of turbulent viscosity

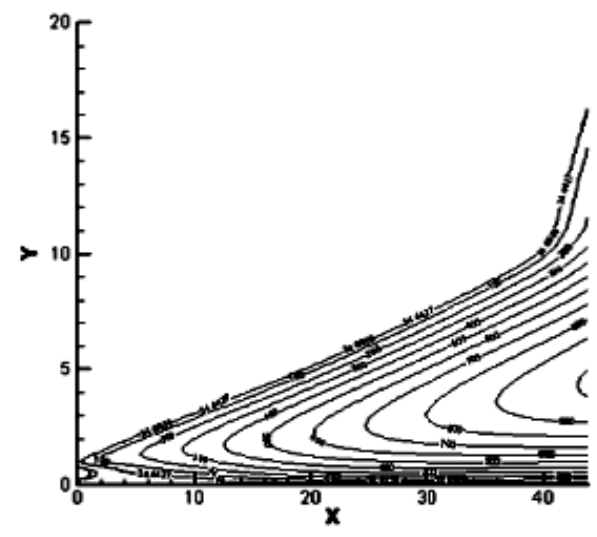

(a)

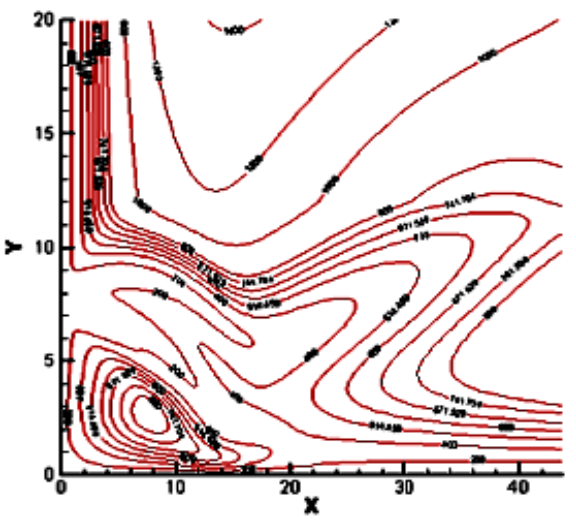

(b)

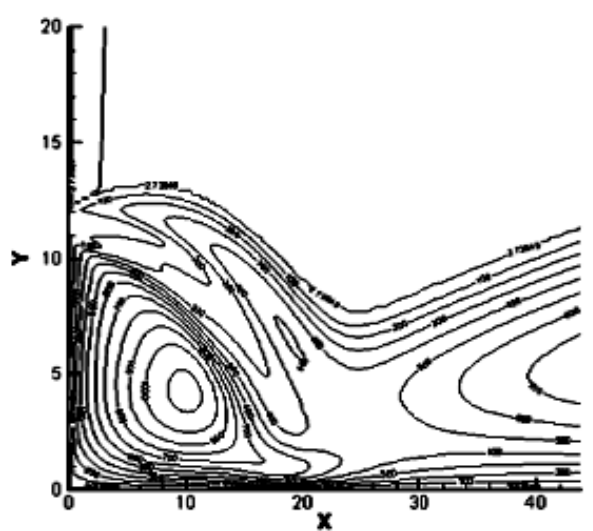

(c)

Figure11. Turbulent viscosity distribution of Offset jet at different offset ratios 
It is maximum at the center of the recirculating region, slightly decreases in the impingement region and then increases in the wall jet region shown in Fig(11). It increases up to $U_{\max }$ in the crosswise direction then decreases. Outside the boundary layer its value s very high which shows high viscous flow in this region. At the center of vortex it's value is very high and it increases as the offset ratio increases at this location. Farther downstream it increases from impingement region to wall jet region.

\section{CONClusion}

- Reattachment length increases with increasing o_set ratio and the variation is of linear type.

- Minimum value of $U_{\max }$ occurs very near to reattachment point in downstream location and it decreases as offset ratio increases.

- In the recirculating region shear stress is positive and it decreases as offset ratio increases.

- Pressure distribution is nearly constant for wall jet region.

\section{Nomenclature}

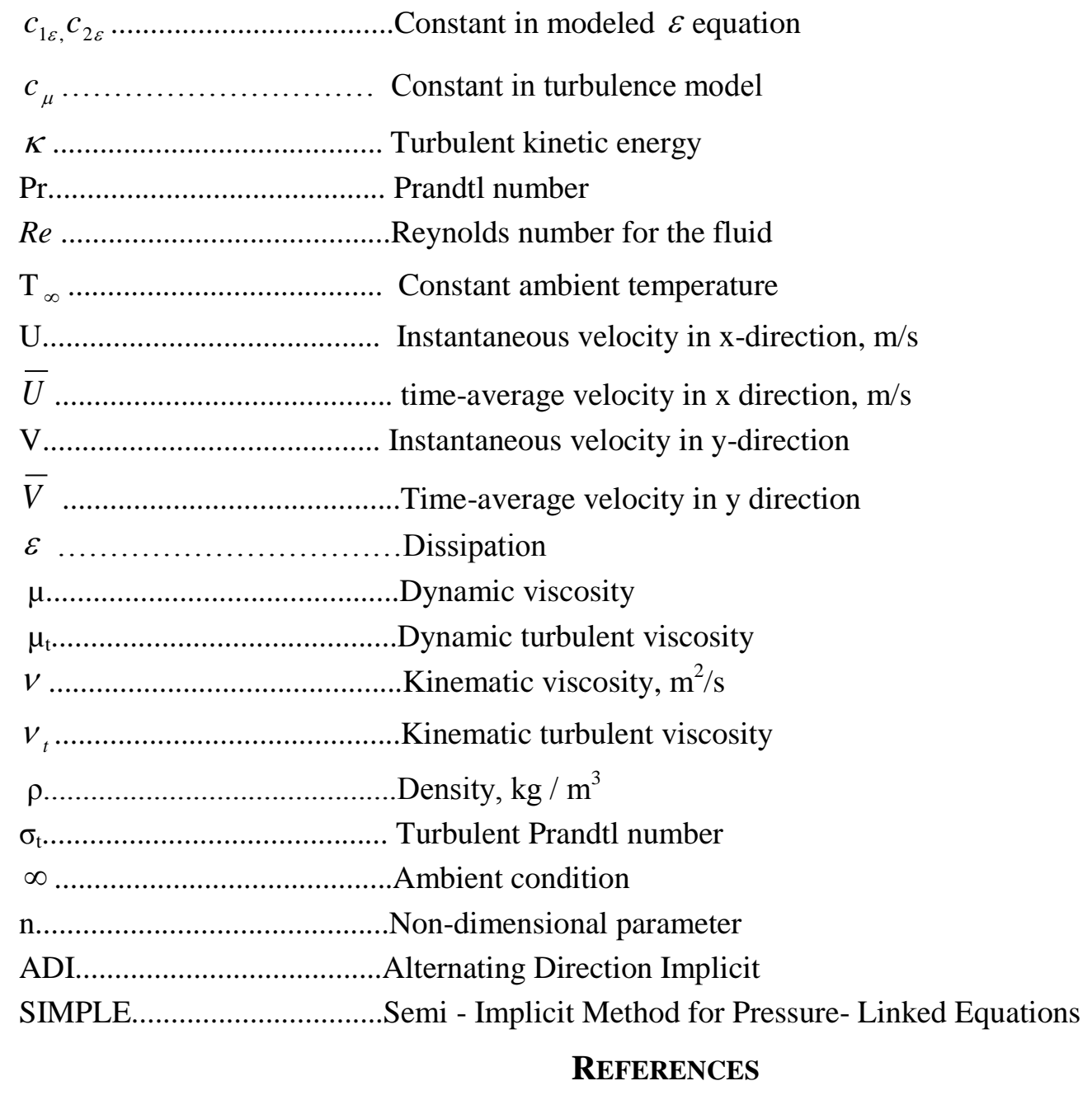

[1] C. Bourque and B.G. Newman, Reattachment of Two - Dimensional Incompressible jet to an Adjacent Plate, Aeronautical Quarterly, vol.11. pp. 201- 232, 1960.

[2] R.A. Sawyer, Two - Dimensional Reattachment jet Flows Including the Effect of Curvature on Entrainment, J. Fluid Mechanics, vol.17, pp. 481 - 498, 1963.

[3] N. Rajratnam and N. Subramanya, Plane Turbulent Reattachment Wall Jets, ASCE J. of Hydraulics Div., vol. 94, no. 1, pp. 95 - 112,1968

[4] J. Hoch and L. M. Jiji, Two - Dimensional Turbulent Offset jet- Boundary Interaction, Transaction ASME J .Fluids Eng., vol. 103, pp. 154-161, 1981.

[5] J. Hoch and L. M. Jiji, Theoretical and Experimental Temperature Distribution in Two Dimensional Turbulent Jet- Boundary Interaction, Transaction ASME J. Heat Transfer, vol. 103, pp. 331-335, 1981. 
[6] J.R.R. Pelfrey and J.A. Liburdy, Mean Flow Characteristics of a Turbulent Offset Jet, Transaction ASME J. Fluids Engineering, vol. 108, pp. 82 - 88, 1986.

[7] J. T. Holland and J. A. Liburdy, Measurements of the Thermal Characteristics of Heated Offset jet, Inter. J. Heat and Mass Transfer, vol. 33, no. 1, pp. 69 - 78, 1990.

[8] S. H. Yoon, S. E. Eun, and M.K. Chung, Numerical Study on the Two - Dimensional Stepped Wall jet, Trans. Korean Society of Mechanical Eng., vol.12, pp. 865 - 875, 1988A.

[9] H. M. Koo and S.O. Park, Prediction of Turbulent Offset Jet Flows with an Assessment of QUICKER Scheme, Inter. J. Numerical. Methods in Fluids, vol. 15, pp. 355 - 372, 1992.

[10] Nasr and J.C.R. Lai, A Turbulent Plane Offset Jet with small Offset Ratio, Exper. in Fluids, vol. 24, pp. $47-57,1998$.

[11] Y. T. Yang and C. H. Shyu, Numerical Study of Multiple Impinging Slot jets with an Inclined Confinement Surface, Numerical. Heat Transfer A, vol. 33, no. 1, pp. 23 - 37, 1998.

[12] S. Z. Shuja, B. L. Yilbas, and M. O. Budair, Gas Jet Impingement on a Surface Having a Limited Constant Flux Area: Various Turbulence Models, Numerical. Heat Transfer A, vol. 36, no. 2, pp. 171 - 200, 1999.

[13] L. B. Y. Aldabbagh and I. Sezai, Numerical Simulation of Three- Dimensional Laminar, Square Twin- Jet Impingement on a Flat Plate, Flow Structure and Heat Transfer, Numerical. Heat Transfer A, vol. 41, no. 8, pp. 835-850, 2002.

[14] S. A. Salamah and D.A. Kaminski, Modeling of Turbulent Heat Transfer from an Array of Submerged Jets Impinging on a Solid Surface, Numerical. Heat Transfer A, vol. 48, no. 4, pp. 315- 337, 2005.

[15] P.R. Kanna and M.K. Das, Conjugate Heat Transfer Study of Two - Dimensional Laminar Incompressible Offset Jet Flows, Numerical. Heat Transfer A. vol. 48, no. 7, pp. 671 -691, 2005

\section{AUTHOR's BIOGRAPHY}

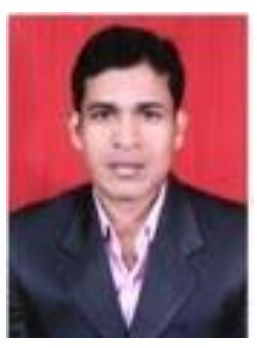

Dr. Ramesh Chandra Mohapatra, was born in Orissa, India in 1969 . He graduated from Department of Mechanical Engineering of UCE, Burla, now VSSUT, Burla, Orissa, India. He received the degree of M.Tech in Thermal Engineering from Department of Mechanical Engineering of Indian Institute of Technology (IIT), Kharagpur, Orissa, India in 2011 and $\mathrm{PhD}$ in Mechanical Engineering from Utkal university, Vanivihar, Bhubaneswar, Orissa, India. He is presently working as Reader \& HOD of Mechanical Engineering Department in Government College of Engineering, Keonjhar, Orissa, India 